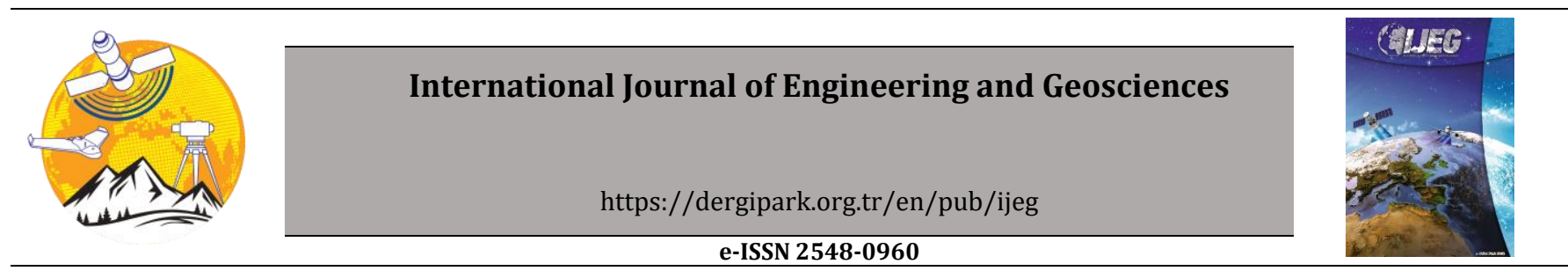

\title{
A new color distance measure formulated from the cooperation of the Euclidean and the vector angular differences for lidar point cloud segmentation
}

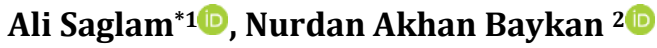 \\ ${ }^{1}$ Konya Technical University, Engineering and Natural Sciences Faculty, Department of Computer Engineering, Konya, Turkey
}

\section{Keywords}

Point Cloud Segmentation

Weighting

Color Distance

Measurement Vector

Angular Difference

\begin{abstract}
Two important features of the points in the LiDAR point clouds are the spatial and the color features. The spatial feature is mostly used in the point cloud processing field due to its 3D informative and distinctive characteristic. The local geometric difference derived from the spatial features of the points is usually benefited by graph-based point cloud segmentation methods, because the geometric features of the local point groups are highly distinctive. In this paper, we use both the geometric and color differences of the adjacent local point groups at the impact rates $0.3,0.5$, and 0.7 and cooperate the Euclidean and the vector color differences within several averaging techniques for the color difference. The difference forms have been tested within a graph-based segmentation method on four point cloud segmentation datasets, two indoor and two outdoor, using their spatial and color information. The geometric mean as an averaging techniques increases the segmentation success for the all datasets except one outdoor when the color differences are used in the segmentation at the impact rate 0.3 , while the harmonic mean increases the success for the all datasets the successes except the other outdoor at the same impact rate. According to the test results, the cooperating of the Euclidean and vector angular color difference measurements can considerable increase the segmentation success on the point clouds with color information in a high quality.
\end{abstract}

\section{INTRODUCTION}

Point clouds are 3D spatial and usually colored data obtained with light detection and ranging systems (LiDAR) (Strom et al., 2010). Because the points in a LiDAR point cloud inherently come as high amount and unorganized, to extract meaningful information from the data is a challenging problem (Li et al., 2017). The point cloud segmentation, which groups the points to reduce the data to be processed and extract new features, is an intermediate stage through the process of extracting meaningful information (Barnea and Filin, 2013).

Graph-based segmentation methods are widely preferred to segment the data for the segmentation process in both the image and point cloud processing fields. An efficient graph-based method (EGS) proposed for 2D image segmentation in (Felzenszwalb and Huttenlocher, 2004) is a widespread segmentation method due to the fastness and segmentation success. In this method, the nearby vertices (elements) are assumed to be connected by weighted edges. The weight values are weighted considering the differences in the color values between pixels for the 2D image segmentation process. Because the structures of 3D point clouds are unorganized unlike 2D images, it is difficult to process 3D point clouds. To deal with this problem, the points are usually grouped into regular 3D volumes (voxels) by the octree organization (Su et al., 2016; Xu et al., 2017). In this way, the edges are assumed between the adjacent voxels.

On the other hand, it is an advantage for the point cloud processing field that LiDAR data has 3D spatial features. The 3D feature provides geometrical features which are more distinctive according to the color feature. Therefore, most graph-based point cloud segmentation methods use the local surface orientation difference for weighting the connections instead of color difference (Vo et al., 2015; Xu et al., 2018a). The other reason for the preference of geometrical differences is that the color feature is usually misleading because the color 
information of the points is sensitive to the brightness varying according to the light and the reflectiveness varying according to the materials of objects (Xu et al., 2018a). Another problem is that some spatially adjacent objects have similar colors. Nevertheless, some methods allow users to use color information with an adjustable influence rate, while some use color information at a certain rate (Papon et al., 2013; Strom et al., 2010; Zhu et al., 2017).

The quality of the color information of point clouds varies and quite affects the success of the segmentation stage if the color information is used at the rate that is higher than 0. In this work, we enforce to increases the segmentation success by using the color information more efficiently. In this reason, we focus on the local color differences methods between two adjacent elements in the RGB (red, green, and blue) color space. In the literature, the Euclidean distance is widely used for the color difference between the two elements (Aijazi et al., 2013; Bassier et al., 2017; Dutta et al., 2014; Papon et al., 2013; Strom et al., 2010). On the other hand, Chen et al. 2019 use the spectral angle as color difference in their study. In this work, we use the vector angular difference and cooperate with the Euclidean distance in some forms. The tested cooperation forms consist of the arithmetic, geometric and harmonic means of the two measurements.

Through our experiments, two indoor coarse and two outdoor fine point cloud segmentation datasets that include the RGB (red, green, and blue) color information of the points and reference segments. The color measures have been tested on the method EGS at the intervals $0.3,0.5$, and 0.7 . The graphical results for two datasets are shown in the experimental results. As a quantitative segmentation evaluation, the Accuracy success measurement (Polak et al., 2009) has been used by looking at the compatibility between the result and reference segments after pairing them mutually one-toone. According to the Accuracy values, the cooperated color difference measurements increases the segmentation success at the influence rate 0.3 for nearly all of the tested datasets.

The main contribution of this study is to present a new approach on the color distance measure for color informed point cloud segmentation. The result show that the cooperating of the Euclidean and vector angular differences by averaging as geometric and harmonic can significantly increases the segmentation success when the color information is a high quality in point clouds.

\section{METHOD}

\subsection{Voxelization}

Voxels are equal-sized 3D cubic volumes and of regular/organized data structures (Lohmann, 1998). For the voxel organization, the octree data structure has been used in this study like many studies in the literature. The octree organization is performed through dividing the volumes into eight equal-sized sub-volumes by starting from the sup-volume that covers all points in the point cloud and specified according to the desired voxel size until the sub-volumes reach the intended voxel size. The voxel size refers to the length of an edge of the voxels. Through the voxelization process, the points are appointed into the voxels according to spatial coordinates.

\subsection{EGS Segmentation Method}

Felzenszwalb and Huttenlocher, 2004 proposed a new segmentation method, named as "Efficient GraphBased Method" (EGS), in 2004. The method runs successfully on 2D images in an effectively short time. The method regards the data elements (pixels for 2D images) as vertices and the connection between the adjacent vertices as edges. The edges are weighted with the Euclidean distance between the color vectors of the two vertices that are ends of the edges. In Fig. (1), the stages of the segmentation is presented.

According to the method, at first, each element is seen as a segment and has a unique segment label. The edges are sorted in ascending order according to their weight values. Beginning from the smallest edges, the edges are considered to remove from the graph with respect to the criterion in Eq. (1). If the weight value $w(u, v)$ of the edge between the vertices $u$ and $v$ meets the criteria, the edge is removed from the graph. Otherwise, the segments at the ends of the edge are involved in the same segments, and the elements in the two segments are labeled with the same segment label. If the segment labels are already the same, the edge is not evaluated by the criterion and directly removed. In Eq. (1), $\operatorname{Int}(u)$ and $\operatorname{Int}(v)$ are the longest edge in the segments $S_{u}$ and $S_{v}$, respectively. $\left|S_{u}\right|$ and $\left|S_{v}\right|$ refer to the number of elements in the segments $S_{u}$ and $S_{v}$, respectively. The parameter $k$ determines the degree of segmentation (under-segmentation or oversegmentation).

$$
w(u, v)>\min \left(\operatorname{Int}(u)+\frac{k}{\left|S_{u}\right|}, \operatorname{Int}(v)+\frac{k}{\left|S_{v}\right|}\right)
$$

\subsection{Geometric Difference}

The most used feature of the point groups in the voxels is the surface normals (Rabbani et al., 2006). The normal vectors give the inclination of the local 3D surfaces and the PCA (Principal Component Analyses) method is the most used technique to obtain it (Lari and Habib, 2014). The angle between the normals of two adjacent voxels is one of the basic geometric differences. The normalized form $\widetilde{\boldsymbol{d}_{\boldsymbol{u}}}$ of the vector $\boldsymbol{d}_{\boldsymbol{u} v}$ between the spatial centers $\boldsymbol{X}_{\boldsymbol{u}}$ and $\boldsymbol{X}_{\boldsymbol{v}}$ of two-point groups is a way to estimate the orientation through two adjacent local surfaces (Stein et al., 2014; Verdoja et al., 2017; Xu et al., 2018a). The angles $\alpha_{u}$ and $\alpha_{v}$ between the vector $\widetilde{\boldsymbol{d}_{\boldsymbol{u} v}}$ and the normals $\widetilde{\boldsymbol{n}_{\boldsymbol{u}}}$ and $\widetilde{\boldsymbol{n}_{\boldsymbol{v}}}$ used widely to measure a geometric difference from the two local surfaces. As the geometric difference $D_{u v}^{G}$ in this work, we have used the formula in Eq. (2).

$$
D_{u v}^{G}=\frac{\alpha_{u}+\alpha_{v}}{2}
$$


The angles $\boldsymbol{\beta}_{\boldsymbol{u}}$ and $\boldsymbol{\beta}_{\boldsymbol{v}}$ in Fig. 2 (a) and Fig. 2 (b) are the acute angles between the normals and the vector $\widetilde{\boldsymbol{d}_{u v}}$, namely, the angles can be in the range $0-90^{\circ}$. The angles $\boldsymbol{\alpha}_{\boldsymbol{u}}$ and $\boldsymbol{\alpha}_{\boldsymbol{v}}$ are obtained by subtracting the angles $\boldsymbol{\beta}_{\boldsymbol{u}}$ and $\boldsymbol{\beta}_{v}$ from $90^{\circ}$.

\subsection{Color Difference}

LiDAR systems can integrate the spatial and color information about the scanned surfaces. In this way, the points in a point cloud save the values of both the coordinate and color vectors. The color information exists generally as RGB values. Each RGB color vector denotes a vector in the RGB color space.

In the graph-based segmentation methods, the Euclidean distance between two color vectors is the most used measurement technique to measure the similarity/dissimilarity. To weight the edges between the vertices (voxels) with the Euclidean distance in point clouds, the mean RGB color values $\left(u^{R}, u^{G}\right.$ and $\left.u^{B}\right)$ of the points in the voxel $u$ are used. The Euclidean distance $E_{u v}^{R G B}$ for color differences between the adjacent voxels $u$ and $v$ can be calculated with Eq. (3) and seen in Fig. 3.

$$
E_{u v}^{R G B}=\sqrt{\left(u^{R}-v^{R}\right)^{2}+\left(u^{G}-v^{G}\right)^{2}+\left(u^{B}-v^{B}\right)^{2}}
$$

The vector angular difference is another distance measurement technique between two vectors as seen in Fig. 3. The angular difference $\hat{A}_{u v}^{R G B}$ between two color vectors $\boldsymbol{u}^{\boldsymbol{R} \boldsymbol{G} \boldsymbol{B}}$ and $\boldsymbol{v}^{\boldsymbol{R} \boldsymbol{G} \boldsymbol{B}}$ can be in the range $0-.90^{\circ}$. The greater the angular difference, the greater the color tone difference. If the angular difference is small but the vector length difference is high between two RGB color vectors, their color tone is similar but color lightness/darkness is different. This case allows the varying brightness over the surfaces to be ignored in the color-supported segmentation process.

In this work, the values of the scaled Euclidean and vector angular differences, and their different cooperated forms in Table 1 are tested, with the different influence rates, to weight values of edges. To evaluate both the color differences within the equal range, the calculated Euclidean distance $\boldsymbol{E}^{\boldsymbol{R} \boldsymbol{G} \boldsymbol{B}}$ are scaled to the range 0-90 like the vector angular difference $\widehat{A}^{R G B}$ and the geometric difference $\boldsymbol{D}^{\boldsymbol{G}}$. In the normalization operation for the scaled Euclidean distance $\widehat{\boldsymbol{E}}^{\boldsymbol{R} \boldsymbol{G} \boldsymbol{B}}$, the lower and upper limits were considered as 0 and the maximum distance among the calculated Euclidean distance values.

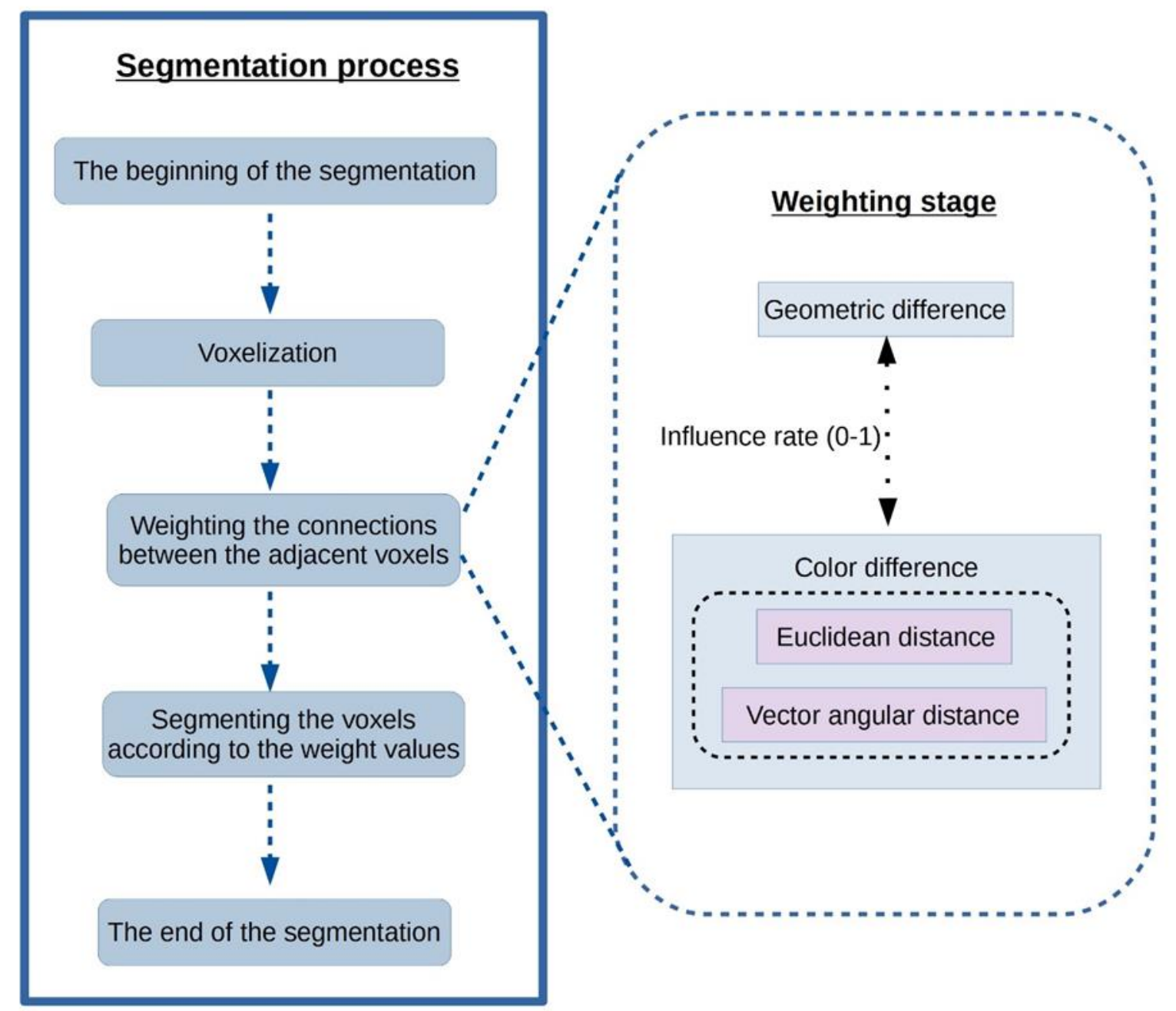

Figure 1. The weighting stage in the segmentation 


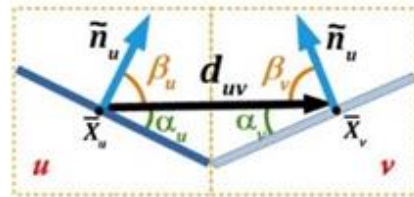

(a)

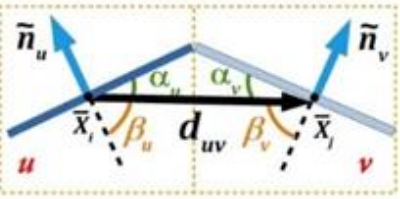

(b)
Figure 2. Some geometric differences between two local surfaces

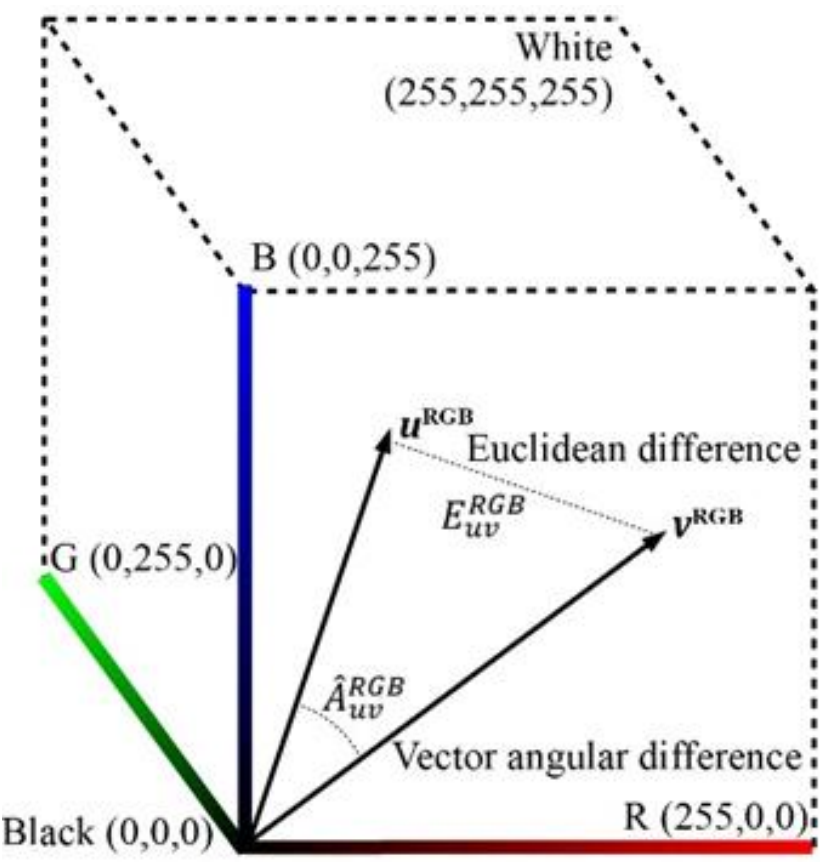

Figure 3. The Euclidean and vector angular differences between two color vectors

Table 1. Cooperation forms of color differences

\begin{tabular}{lc}
\hline Name & Color Difference Form \\
\hline Euclidean color difference & $\hat{E}^{R G B}$ \\
Vector angular color & $\hat{A}^{R G B}$ \\
difference & $\frac{\hat{E}^{R G B}+\hat{A}^{R G B}}{2}$ \\
Arithmetic mean & $\sqrt{\hat{E}^{R G B} \times \hat{A}^{R G B}}$ \\
Geometric mean & $2 \times \frac{\hat{E}^{R G B} \times \hat{A}^{R G B}}{\hat{E}^{R G B}+\hat{A}^{R G B}}$ \\
\hline
\end{tabular}

\section{EXPERIMENTAL RESULTS}

\subsection{Datasets}

To test the color difference forms, the forms have been tested on four sample point cloud datasets (two indoors which are named as "Indoor 1" and "Indoor 2", and two outdoors which are named as "Outdoor 1 " and "Outdoor 2"). The indoor datasets are coarse segmentation datasets prepared for segmantic segmentation by (Armeni et al., 2016), while the outdoor datasets are cropped from outdoor building scans, which have been prepared from the two large-scale point cloud classification benchmark datasets (Hackel et al., 2017) with reference segments by (Xu et al., 2018a) and used by permission of (Xu et al., 2018b). The test data are shown with their original RGB colors in Fig. 4.

\subsection{Results}

In our experiments, the color difference forms in Table 1 have been used at the influence rates $0.3,0.5$, and 0.7 with the geometric difference in Eq. (2) for the weight values of edges of the EGS method with the segmentation parameter $k$ in the range 0-300 with 10 intervals. The voxel size was specified as 0.1 like the study (Xu et al., 2018a).

As a quantitative evaluation, Accuracy measurement is used (Saglam and Baykan, 2019). According to the Accuracy measurement for segmentation success, the result segments and the reference segments have been paired firstly one-to-one, mutually. The pairing process is carried out according to the study (Awrangjeb and Fraser, 2014). After the pairing process, some segments among the result and reference segments may not be paired with any mutual segments. The ratio of the number of common points in the segment pairs to the number of points in the reference data indicates the Accuracy value.

In Table 2, the Accuracy results of the segmentation results with the best $k$ parameter according to the color difference forms at the influence rates $0.3,0.5$, and 0.7 are taken part. In Fig. 5, the colored presentations of some segmentation results with reference data are demonstrated.

\section{CONCLUSION}

The process of point cloud segmentation is an important intermediate stage to extract meaningful information from the raw point clouds. The spatial geometric features are the most used property for graphbased point cloud segmentation methods. In this paper, we have added the color influence to local dissimilarity to weight connections in the graph structure at several influence rate. In the weighting method, the Euclidean and vector angular color differences are cooperated in some forms as arithmetic mean, geometric mean and harmonic means. The test was carried out on two indoor and two outdoor datasets using an efficient graph-based segmentation method. The method that cooperates the two color difference measures, especially the geometric mean and the harmonic mean, has substantially increased the segmentation success on the indoor datasets. On the other hand, the successes on the outdoor datasets is increased slightly because of the lack of color information on the outdoor datasets. The geometric mean as an averaging techniques increases the segmentation success for the all datasets except one outdoor when the color differences are used in the segmentation at the impact rate 03 , while the harmonic mean increases the success for the all datasets the successes except the other outdoor at the same impact rate. The results show that the method is very useful when the segmentation are processed on the point clouds with sufficient color information.

\section{ACKNOWLEDMENT}

This study was carried out in the scope of the Doctoral Thesis of Ali SAGLAM. 


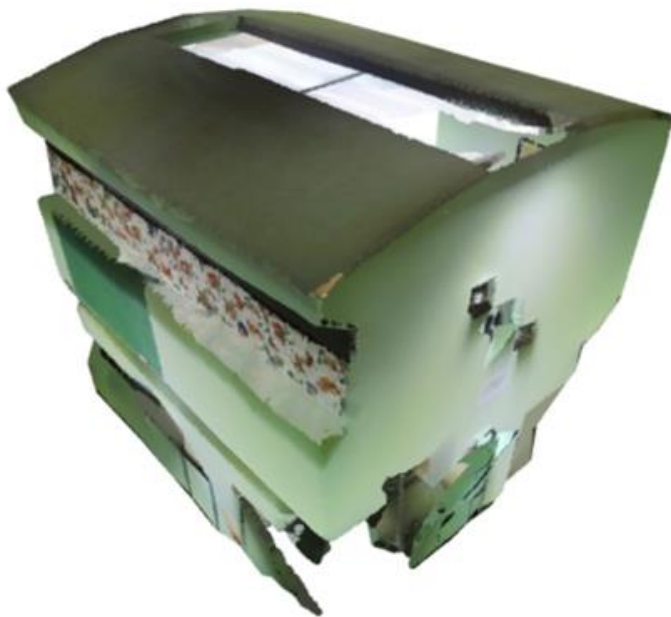

Indoor 1

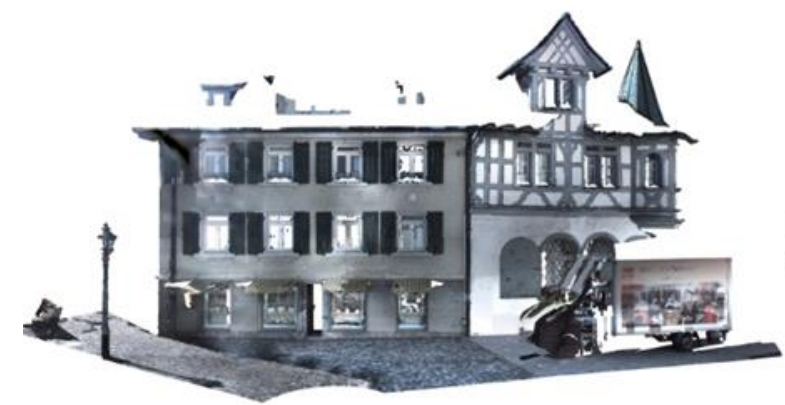

Outdoor 1

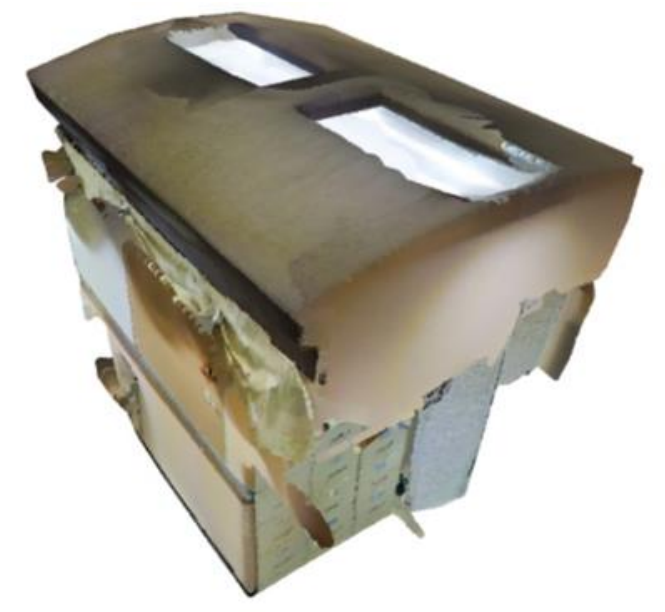

Indoor 2

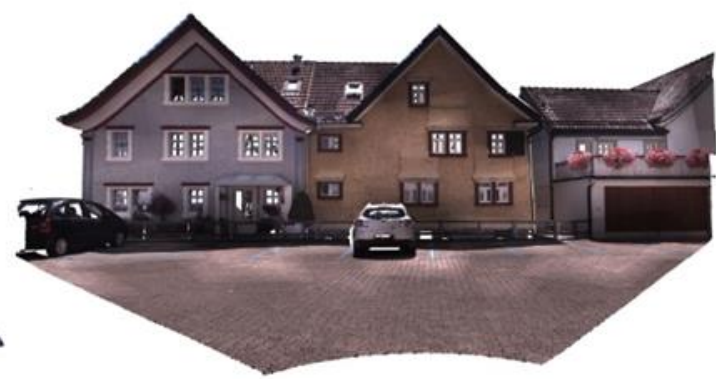

Outdoor 2

Figure 4. The original versions of the datasets used for segmentation

Table 2. Accuracy results of the colour difference forms

\begin{tabular}{lcllcc}
\hline \multirow{2}{*}{ Color difference form } & \multirow{2}{*}{ Color influence rate } & \multicolumn{3}{c}{ Dataset } \\
\cline { 3 - 6 } No color & 0 & Indoor 1 & Indoor 2 & Outdoor 1 & Outdoor 2 \\
\hline Scaled Euclidean difference & 0.3 & 0.6997 & 0.7187 & 0.6879 & 0.8036 \\
\hline Scaled Euclidean difference & 0.5 & 0.7057 & 0.7528 & 0.6076 & 0.7820 \\
Scaled Euclidean difference & 0.7 & 0.7093 & 0.7158 & 0.5718 & 0.7209 \\
Vector angular difference & 0.3 & 0.6641 & 0.7058 & 0.4792 & 0.7058 \\
Vector angular difference & 0.5 & 0.6807 & 0.7232 & 0.6593 & 0.7847 \\
Vector angular difference & 0.7 & 0.7154 & 0.7876 & 0.6928 & 0.7507 \\
\hline Arithmetic mean & 0.3 & 0.7080 & 0.7588 & 0.6384 & 0.6968 \\
Arithmetic mean & 0.5 & 0.6931 & 0.6873 & 0.6225 & 0.7810 \\
Arithmetic mean & 0.7 & 0.7282 & 0.7643 & 0.5564 & 0.7342 \\
\hline Geometric mean & 0.3 & 0.6579 & 0.7484 & 0.4912 & 0.6868 \\
Geometric mean & 0.5 & 0.7094 & 0.7937 & 0.6849 & 0.8176 \\
Geometric mean & 0.7 & 0.7351 & 0.7566 & 0.6294 & 0.7960 \\
\hline Harmonic mean & 0.3 & 0.7077 & 0.7153 & 0.5703 & 0.7168 \\
Harmonic mean & 0.5 & 0.7048 & 0.7436 & 0.7132 & 0.7782 \\
Harmonic mean & 0.7 & 0.7884 & 0.7386 & 0.6770 & 0.7787 \\
\hline
\end{tabular}




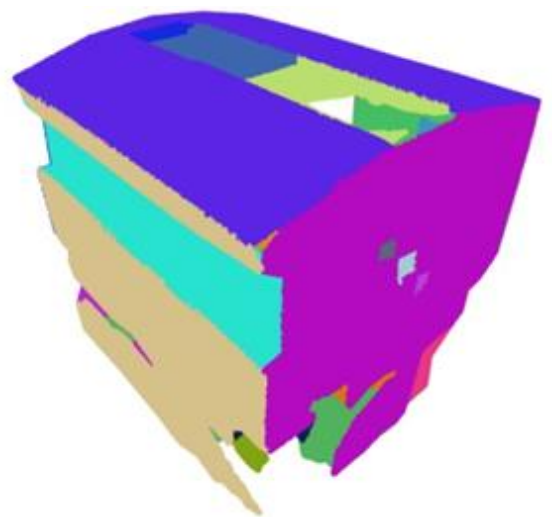

Indoor 1 Reference

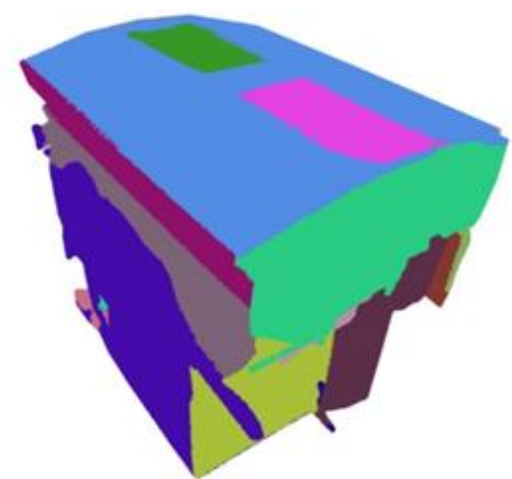

Indoor 2 Reference

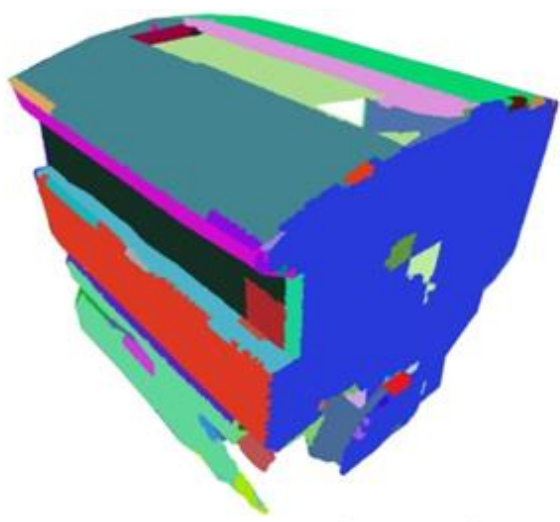

Indoor 1 Result (no color)

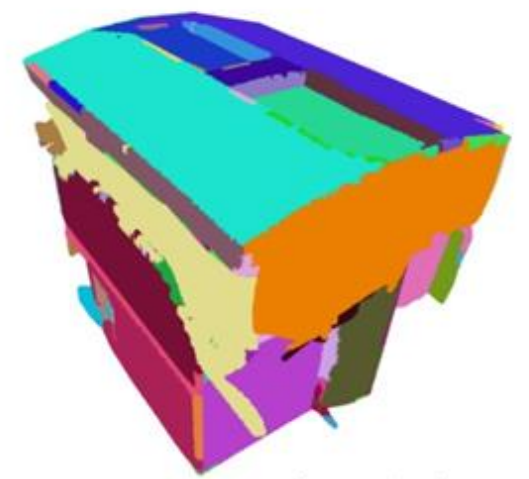

Indoor 2 Result (no color)

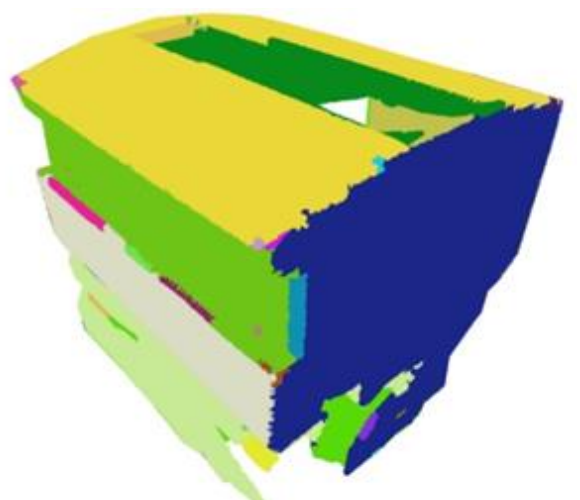

Indoor 1 Result

(Harmonic Mean - IR=0.5)

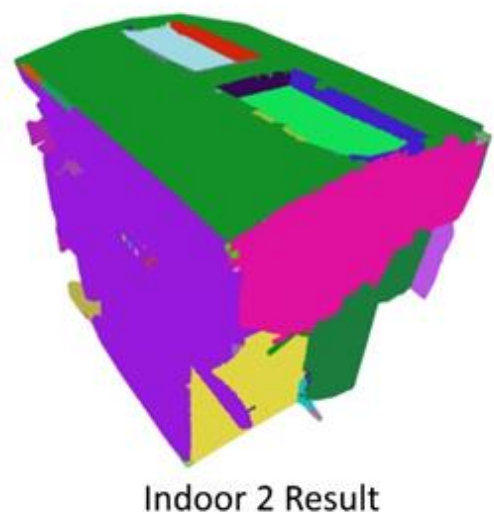

(Geometric Mean - IR=0.3)
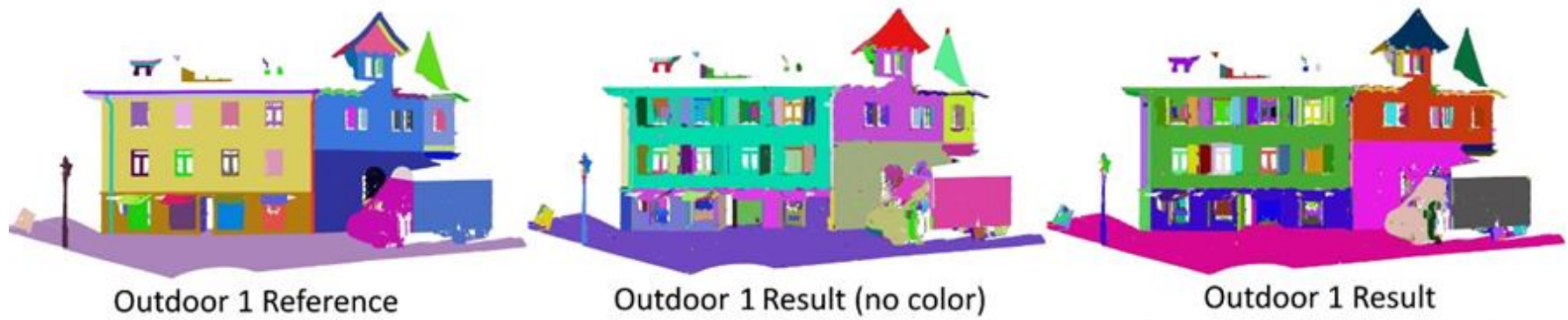

(Harmonic Mean - IR=0.3)

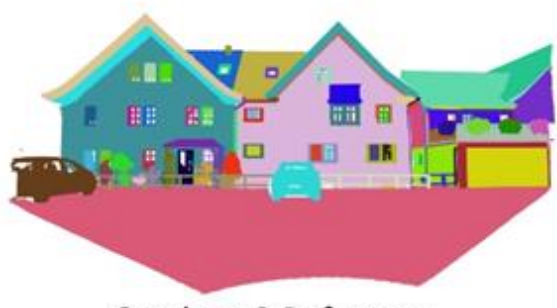

Outdoor 2 Reference

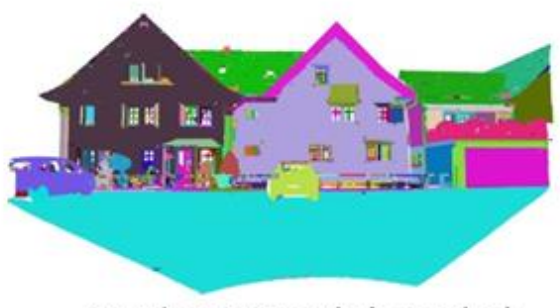

Outdoor 2 Result (no color)

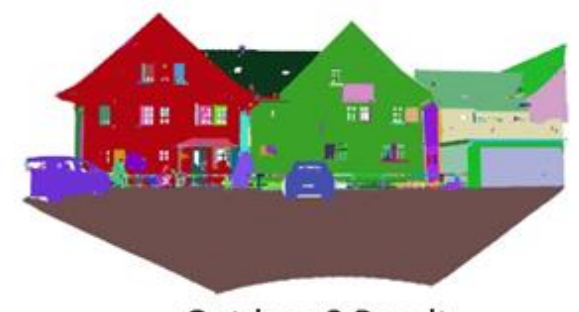

Outdoor 2 Result

(Geometric Mean - IR=0.3)

Figure 5. The colored reference data and segmentation results (with no color and some cooperated forms) 


\section{REFERENCES}

Aijazi A K, Checchin P \& Trassoudaine L (2013). Segmentation Based Classification of 3D Urban Point Clouds: A Super-Voxel Based Approach with Evaluation. Remote Sensing, 5(4), 1624-1650. https://doi.org/10.3390/rs5041624

Armeni I, Sener O, Zamir A R, Jiang H, Brilakis I, Fischer M \& Savarese S (2016). 3D semantic parsing of largescale indoor spaces. Proceedings of the IEEE Computer Society Conference on Computer Vision and Pattern Recognition. https://doi.org/10.1109/CVPR.2016.170

Awrangjeb M \& Fraser C S (2014). An automatic and threshold-free performance evaluation system for building extraction techniques from airborne LIDAR data. IEEE Journal of Selected Topics in Applied Earth Observations and Remote Sensing 7(10), 4184-4198. https://doi.org/10.1109/JSTARS.2014.2318694

Barnea S \& Filin S (2013). Segmentation of terrestrial laser scanning data using geometry and image information. ISPRS Journal of Photogrammetry and Remote Sensing, 76, 33-48. https://doi.org/10.1016/j.isprsjprs.2012.05.001

Bassier M, Bonduel M, Van Genechten B \& Vergauwen M (2017). Segmentation of large unstructured point clouds using octree-based region growing and conditional random fields. International Archives of the Photogrammetry, Remote Sensing and Spatial Information Sciences - ISPRS Archives XLII-2/W8, 25-30. https://doi.org/10.5194/isprs-archives-XLII2-W8-25-2017

Chen B, Shi S., Sun J, Gong W, Yang J, Du L, Guo K, Wang, B, Chen, B (2019). Hyperspectral lidar point cloud segmentation based on geometric and spectral information. Optics Express, 27(17). https://doi.org/10.1364/oe.27.024043

Dutta A, Engels J \& Hahn M (2014). A distance-weighted graph-cut method for the segmentation of laser point clouds. International Archives of the Photogrammetry, Remote Sensing and Spatial Information Sciences - ISPRS Archives 40(3), 81-88. https://doi.org/10.5194/isprsarchives-XL-3-812014

Felzenszwalb P F \& Huttenlocher D P (2004). Efficient graph-based image segmentation. International Journal of Computer Vision, 59(2), 167-181. https://doi.org/10.1023/B:VISI.0000022288.19776. 77

Hackel T, Savinov N, Ladicky L, Wegner J D, Schindler K \& Pollefeys M (2017). Semantic3D.net: A new Largescale Point Cloud Classification Benchmark. ISPRS Annals of the Photogrammetry, Remote Sensing and Spatial Information Sciences. https://doi.org/10.5194/isprs-annals-IV-1-W1-912017

Lari Z \& Habib A (2014). An adaptive approach for the segmentation and extraction of planar and linear/cylindrical features from laser scanning data. ISPRS Journal of Photogrammetry and Remote Sensing, 93,

192-212. https://doi.org/10.1016/j.isprsjprs.2013.12.001
Li L, Yang F, Zhu H, Li D, Li Y \& Tang L (2017). An improved RANSAC for 3D point cloud plane segmentation based on normal distribution transformation cells. Remote Sensing 9(5). https://doi.org/10.3390/rs9050433

Lohmann G (1998). Volumetric image analysis. Wiley.

Papon J, Abramov A, Schoeler M \& Worgotter F (2013). Voxel cloud connectivity segmentation - Supervoxels for point clouds. Proceedings of the IEEE Computer Society Conference on Computer Vision and Pattern Recognition, 2027-2034. https://doi.org/10.1109/CVPR.2013.264

Polak M, Zhang H \& Pi M (2009). An evaluation metric for image segmentation of multiple objects. Image and Vision Computing, 27(8), 1223-1227. https://doi.org/10.1016/j.imavis.2008.09.008

Rabbani T, van den Heuvel F A \& Vosselman G (2006). Segmentation of point clouds using smoothness constraint. International Archives of Photogrammetry, Remote Sensing and Spatial Information Sciences - Commission V Symposium "Image Engineering and Vision Metrology" 36, 248253. https://doi.org/10.1111/1750-3841.12802

Saglam A \& Baykan N A (2019). Evaluating the attributes of remote sensing image pixels for fast k-means clustering. Turkish Journal of Electrical Engineering \& Computer Sciences, 27, 4188-4202. https://doi.org/10.3906/elk-1901-190

Stein S C, Schoeler M, Papon J \& Worgotter F (2014). Object partitioning using local convexity. Proceedings of the IEEE Computer Society Conference on Computer Vision and Pattern Recognition, 304-311. https://doi.org/10.1109/CVPR.2014.46

Strom J, Richardson A \& Olson E (2010). Graph-based segmentation for colored 3D laser point clouds. IEEE/RSJ 2010 International Conference on Intelligent Robots and Systems, 2131-2136. https://doi.org/10.1109/IROS.2010.5650459

Su Y T, Bethel J \& Hu S (2016). Octree-based segmentation for terrestrial LiDAR point cloud data in industrial applications. ISPRS Journal of Photogrammetry and Remote Sensing 113, 59-74. https://doi.org/10.1016/j.isprsjprs.2016.01.001

Verdoja F, Thomas D \& Sugimoto A (2017). Fast 3D point cloud segmentation using supervoxels with geometry and color for 3D scene understanding. Proceedings IEEE International Conference on Multimedia and Expo, Hong Kong, China, 1285-1290. https://doi.org/10.1109/ICME.2017.8019382

Vo A V, Truong-Hong L, Laefer D F \& Bertolotto M (2015). Octree-based region growing for point cloud segmentation. ISPRS Journal of Photogrammetry and Remote Sensing 104, 88-100. https://doi.org/10.1016/j.isprsjprs.2015.01.011

Xu Y, Hoegner L, Tuttas S \& Stilla U (2017). Voxel- and graph-based point cloud segmentation of 3D scenes using perceptual grouping laws. ISPRS Annals of Photogrammetry, Remote Sensing and Spatial Information Sciences IV-1/W1, 43-50. https://doi.org/10.5194/isprs-annals-IV-1-W1-432017

Xu Y, Yao W, Tuttas S, Hoegner L \& Stilla U (2018a). Unsupervised Segmentation of Point Clouds From 
Buildings Using Hierarchical Clustering Based on Gestalt Principles. IEEE Journal of Selected Topics in Applied Earth Observations and Remote Sensing 11, 4270-4286.

https://doi.org/10.1109/JSTARS.2018.2817227

Xu Y, Yao W, Tuttas S, Hoegner L \& Stilla U (2018b). Building-Segmentation-Reference-Dataset [WWW Document]. URL https://github.com/Yusheng$\mathrm{Xu} /$ Building-Segmentation-Reference-Dataset
Zhu Q, Li Y, Hu H \& Wu B (2017). Robust point cloud classification based on multi-level semantic relationships for urban scenes. ISPRS Journal of Photogrammetry and Remote Sensing, 129, 86-102. https://doi.org/10.1016/j.isprsjprs.2017.04.022

(C) Author(s) 2021.

This work is distributed under https://creativecommons.org/licenses/by-sa/4.0/ 